$46.86 \pm 28.89$ respectively, ð>0.05). After 6 months treatment free T4 levels were lower $(8.5 \pm 5.48)$ in those patients (33.3\%), which undergo higher dosage of thyrostatics and later levothyroxin treatment compared to levothyroxin treatment after 4 months after first appeal $(16.6 \pm 2.92$ ð<0.05). After 1 year treatment with thyrostatics in comparable dosages stable clinical-laboratory euthyrosis were reached in all children.

Conclusions GBD more often manifested in late puberty regardless of gender. More pronounced features of laboratory thyrotoxicosis in boys can say about late diagnostic. Normalization of free T4 was after 6 months treatment in $66.7 \%$ children.

\section{ENHANCEMENT OF LINEAR GROWTH AND WEIGHT VELOCITY BY CYPROHEPTADINE IN CHILDREN WITH IDIOPATHIC GROWTH HORMONE DEFICIENCY RECEIVING THIS HORMONE}

doi:10.1136/archdischild-2012-302724.0656

KS Najib, Z Karamizadeh. Shiraz University of Medical Sciences, Shiraz, Iran

Background The current study examined the hypothesis that Cyproheptadine can enhance linear growth and increase weight velocity in children with idiopathic GH deficiency.

Method 10 children with idiopathic GH deficiency received Cyproheptadine $0.3 \mathrm{mg} / \mathrm{kg}$ three times per week plus GH0.6U/kg/day for six days a week for1-year period, alternatively (GH plus Cyproheptadine for first and third trimesters, and GH plus placebo for second and fourth trimesters). Weight velocity and linear growth were assessed at baselineand at end of every trimester.

Result The repeated measure ANOVA test showed significant differences in weight velocity across the study trimesters so that the increase in weight velocity within first and third trimesters were significantly higher than other time intervals $(\Delta \mathrm{WV}$ : first trimester $1.51 \pm 0.61 \mathrm{~kg}$; second trimester $1.13 \pm 0.46 \mathrm{~kg}$; third trimester $1.87 \pm 0.65 \mathrm{~kg}$; and fourth trimester $0.74 \pm 0.34 \mathrm{~kg}, \mathrm{p}=0.026$ ). Similar trend was also observed regarding changes in linear growth that the increase in children height was significantly higher in first and third trimesters compared with other trimesters $(\Delta \mathrm{LG}$ : first trimester $2.40 \pm 0.39 \mathrm{~cm}$; second trimester $1.65 \pm 0.41 \mathrm{~cm}$; third trimester $2.00 \pm 0.88 \mathrm{~kg}$; and fourth trimester $1.30 \pm 0.48 \mathrm{~kg}, \mathrm{p}=0.029$ ). The standard deviation of linear growth was gradually decreased during the study trimesters $(4.75,4.50,4.25$, and $3.88 \mathrm{~cm}$, respectively, however the changes in this parameter was not different between the two drug regimens.

Conclusion Our study showed improved linear growth and weight velocity following administration of Cyproheptadine in children given $\mathrm{GH}$ because of their GH deficiency.

\section{THE ROLE OF GROWTH HORMONE IN BONE MATURATION: EVALUATION BY HAND X-RAY}

doi:10.1136/archdischild-2012-302724.0657

'L Even, ${ }^{2} \mathrm{Z}$ Hochberg. 'Pediatrics, Bar Ilan Western Gallile Hospital, Naharia; ${ }^{2}$ Technion, Haifa, Israel

The maturation of the long bones and short bones of the hand differs from the carpal bones. We aimed to determine role of $\mathrm{GH}$. Bone age x-ray was performed in 12 children with GHD, 19 ISS children age $5.5 \pm 0.9(\mathrm{~m} \pm \mathrm{sd})$ during $3 \mathrm{y}$ of $\mathrm{hGH}$ treatment and 12 untreated ISS children. Individual bones were evaluated by a single blinded observer according to Greulich and Pyle, and are expressed as 'years' (y) of delay relative to chronological age.

In ISS, maturation was delayed by $1.87 \pm 0.3,2.07 \pm 0.25$ and $1.75 \pm 0.2$ ' $y$ ' for RU, C and S bones, resp. In GHD, maturation was delayed by $3.1 \pm 0.4,4.5 \pm 0.2$ and $2.9 \pm 0.4$ ' $y$ ' for RU, $C$ and $S$ bones, resp. In ISS over 3 y of GH treatment, RU advanced by a mean $3.5 \pm 0.4$ ' $y$ ', as compared with untreated $3.3 \pm 0.7$ ' $y$ ' $(p<0.10)$,
C advanced by a mean $4.2 \pm 0.7$ ' $y$ ' on $\mathrm{hGH}$ and $3.3 \pm 0.6$ ' $\mathrm{y}$ ', in control $(\mathrm{p}<0.001)$, and $\mathrm{S}$ bones by a mean $3.5 \pm 0.9$ ' $\mathrm{y}$ ' on $\mathrm{hGH}$ and $3.15 \pm 0.7$ ' $y$ ' in control $(p<0.058)$. In GHD over $3 y$ of GH treatment, RU advanced by a mean $3.1 \pm 0.4$ ' $y$ ', $(p<0.10)$, C advanced by a mean $4.3 \pm 0.2$ ' $y$ ' $(p<0.001)$, and $S$ bones $2.9 \pm 0.1(p<0.06)$.

These results suggest that GH strongly regulates and GHD interferes with bone maturation by inhibiting chondral osteogenesis and less so through delayed enchondroplasia, observed by RU and $S$ maturation. These profiles help in the diagnosis of GHD.

\section{CONGENITAL ANOMALIES IN INFANTS WITH CONGENITAL HYPOTHYROIDISM IN OAZVIN, IRAN}

doi:10.1136/archdischild-2012-302724.0658

${ }^{1} \mathrm{~F}$ Saffari, ${ }^{2} \mathrm{Y}$ Melikian. 'Pediatrics, Oazvin University of Medical Sciences; ${ }^{2}$ Oazvin University of Medical Sciences, Oazvin, Iran

Background and Aims In the last decade a high frequency of extrathyroidal congenital anomalies has been reported in infants with congenital hypothyroidism detected by neonatal screening. Aproximately $10 \%$ of infants with congenital hypothyroidism have associated congenital anomalies. Cardiac anomalies are most common, but anomalies of the nervous system and eye have also been reported. The main objective of this study was identification of associated disorders in patients with congenital hypothyroidism.

Methods This descriptive study was performed on newborns with congenital hypothyroidism that had been diagnosed by screening in Qazvin province of Iran from 1385 to 1390 . Variables were analyzed by Chi-square test.

Results In the screening 287 newborns with congenital hypothyroidism were diagnosed -122 female (42.2\%). 36 (12.5\%) of 287 patients had congenital anomalies. 10 (3.5\%) Down syndrome, 10 $(3.5 \%)$ cardiaovascular anomalies, 9 (3.1\%) hearing disorder, 2 $(0.7 \%)$ eye disorder, $2(0.7 \%)$ metabolic disease, $2(0.7 \%)$ disorder of face and ear, $2(0.7 \%)$ CNS disorder, one $(0.3 \%) \mathrm{CAH}$, one $(0.3 \%)$ panhypopituitrism and one (0.3\%) pseudohypoparathyroidism were diagnosed.

Conclusions With respect to the association of congenital anomalies with congenital hypothyroidism, every newborn with congenital hypothyroidism should be carefully examined for cardiaovascular disorders and other related anomalies.

\section{HYPOPARATHYROIDISM AND PSEUDO- HYPOPARATHYROIDIM: ABOUT 9 CASES}

doi:10.1136/archdischild-2012-302724.0659

I Maaloul, S Kmiha, L Sfaihi, I Chabchoub, S Ben Ameur, H Aloulou, T Kamoun, M Hachicha. Pediatric Department, Hedi Chaker Hospital, Sfax, Tunisia

Introduction Hypoparathyroidism(HP) is an uncommon disorder of calcium metabolism characterized by hypocalcemia and hyperphosphatemia due to impaired parathyroid hormone (PTH) secretion. The pseudo-hypoparathyroidism(PHP) is characterized by a high level of PTH due to peripheral PTH resistance.

Objective Describe clinical, biochemical, radiological profile, treatment and outcome in 9 patients with HP and PHP seen over a period of 24 years.

Methods This study was performed in the pediatric department of Sfax during 24 years (from January 1988 to April 2012). The diagnosis of HP and PHP was based on demonstration of hypocalcemia, hyperphosphatemia with low or elevated PTH, respectively.

Results During the period of study, 9 children having HP or PHP were admitted. There were 5 girls and 4 boys. The mean age at presentation was 44 months ( 15 days- 10 years). The most common presenting manifestation was seizures (8cases) followed by carpopedal spasm in one case. The mean serum calcium and inorganic phosphate concentrations were $1,51 \mathrm{mmol} / \mathrm{l}$ and $3 \mathrm{mmol} / \mathrm{l}$, 